\title{
NuSTAR discovery of a luminosity dependent cyclotron line energy in Vela X-1
}

Fuerst, Felix; Pottschmidt, Katja; Wilms, Joern; Tomsick, John A.; Bachetti, Matteo; Boggs, Steven E.; Christensen, Finn Erland; Craig, William W.; Grefenstette, Brian W.; Hailey, Charles J.

Total number of authors:

16

Published in:

Astrophysical Journal

Link to article, DOI:

$10.1088 / 0004-637 X / 780 / 2 / 133$

Publication date:

2014

Document Version

Publisher's PDF, also known as Version of record

Link back to DTU Orbit

Citation (APA):

Fuerst, F., Pottschmidt, K., Wilms, J., Tomsick, J. A., Bachetti, M., Boggs, S. E., Christensen, F. E., Craig, W. W., Grefenstette, B. W., Hailey, C. J., Harrison, F., Madsen, K. K., Miller, J. M., Stern, D., Walton, D., \& Zhang, W. (2014). NuSTAR discovery of a luminosity dependent cyclotron line energy in Vela X-1. Astrophysical Journal, $780(2)$, 133. https://doi.org/10.1088/0004-637X/780/2/133

\section{General rights}

Copyright and moral rights for the publications made accessible in the public portal are retained by the authors and/or other copyright owners and it is a condition of accessing publications that users recognise and abide by the legal requirements associated with these rights.

- Users may download and print one copy of any publication from the public portal for the purpose of private study or research.

- You may not further distribute the material or use it for any profit-making activity or commercial gain

- You may freely distribute the URL identifying the publication in the public portal 


\title{
NuSTAR DISCOVERY OF A LUMINOSITY DEPENDENT CYCLOTRON LINE ENERGY IN VELA X-1
}

\author{
Felix Fürst $^{1}$, Katja Pottschmidt ${ }^{2,3}$, Jörn Wilms ${ }^{4}$, John A. Tomsick ${ }^{5}$, Matteo Bachetti ${ }^{6,7}$, Steven E. Boggs $^{5}$, \\ Finn E. Christensen ${ }^{8}$, William W. Craig ${ }^{5,9}$, Brian W. Grefenstette ${ }^{1}$, Charles J. Hailey ${ }^{10}$, Fiona Harrison ${ }^{1}$, \\ Kristin K. Madsen ${ }^{1}$, Jon M. Miller ${ }^{11}$, Daniel Stern ${ }^{12}$, Dominic J. Walton ${ }^{1}$, And William Zhang ${ }^{13}$ \\ ${ }^{1}$ Cahill Center for Astronomy and Astrophysics, California Institute of Technology, Pasadena, CA 91125, USA \\ ${ }^{2}$ Center for Space Science and Technology, University of Maryland Baltimore County, Baltimore, MD 21250, USA \\ ${ }^{3}$ CRESST and NASA Goddard Space Flight Center, Astrophysics Science Division, Code 661, Greenbelt, MD 20771, USA \\ ${ }^{4}$ Dr. Karl-Remeis-Sternwarte and ECAP, Sternwartstr. 7, D-96049 Bamberg, Germany \\ ${ }^{5}$ Space Sciences Laboratory, University of California, Berkeley, CA 94720, USA \\ ${ }^{6}$ Université de Toulouse, UPS-OMP, IRAP, Toulouse, France \\ ${ }^{7}$ CNRS, Institut de Recherche en Astrophysique et Planétologie, 9 Av. Colonel Roche, BP 44346, F-31028 Toulouse Cedex 4, France \\ ${ }^{8}$ DTU Space, National Space Institute, Technical University of Denmark, Elektrovej 327, DK-2800 Lyngby, Denmark \\ ${ }^{9}$ Lawrence Livermore National Laboratory, Livermore, CA 94550, USA \\ ${ }^{10}$ Columbia Astrophysics Laboratory, Columbia University, New York, NY 10027, USA \\ ${ }^{11}$ Department of Astronomy, The University of Michigan, Ann Arbor, MI 48109, USA \\ 12 Jet Propulsion Laboratory, California Institute of Technology, Pasadena, CA 91109, USA \\ ${ }^{13}$ NASA Goddard Space Flight Center, Astrophysics Science Division, Code 662, Greenbelt, MD 20771, USA \\ Received 2013 September 16; accepted 2013 November 19; published 2013 December 18
}

\begin{abstract}
We present NUSTAR observations of Vela X-1, a persistent, yet highly variable, neutron star high-mass X-ray binary (HMXB). Two observations were taken at similar orbital phases but separated by nearly a year. They show very different 3-79 keV flux levels as well as strong variability during each observation, covering almost one order of magnitude in flux. These observations allow, for the first time ever, investigations on kilo-second time-scales of how the centroid energies of cyclotron resonant scattering features (CRSFs) depend on flux for a persistent HMXB. We find that the line energy of the harmonic CRSF is correlated with flux, as expected in the sub-critical accretion regime. We argue that Vela X-1 has a very narrow accretion column with a radius of around $0.4 \mathrm{~km}$ that sustains a Coulomb interaction dominated shock at the observed luminosities of $L_{\mathrm{x}} \sim 3 \times 10^{36} \mathrm{erg} \mathrm{s}^{-1}$. Besides the prominent harmonic line at $55 \mathrm{keV}$ the fundamental line around $25 \mathrm{keV}$ is clearly detected. We find that the strengths of the two CRSFs are anti-correlated, which we explain by photon spawning. This anti-correlation is a possible explanation for the debate about the existence of the fundamental line. The ratio of the line energies is variable with time and deviates significantly from 2.0, also a possible consequence of photon spawning, which changes the shape of the line. During the second observation, Vela X-1 showed a short off-state in which the power-law softened and a cut-off was no longer measurable. It is likely that the source switched to a different accretion regime at these low mass accretion rates, explaining the drastic change in spectral shape.
\end{abstract}

Key words: accretion, accretion disks - radiation: dynamics - stars: neutron - X-rays: binaries X-rays: individual (Vela X-1)

Online-only material: color figures

\section{INTRODUCTION}

Vela $\mathrm{X}-1$ is one of the most famous wind-accreting neutron star X-ray binaries. The neutron star, rotating with a period of about $283 \mathrm{~s}$ around its spin axis, is in an $8.9 \mathrm{~d}$ orbit around the B0.5Ib super-giant HD 77523 (Hiltner et al. 1972). With an orbital separation of only $1.7 R_{\star}$ (Joss \& Rappaport 1984; Quaintrell et al. 2003), the neutron star is deeply embedded in the stellar wind, accreting on the order of $10^{-9} M_{\odot} \mathrm{yr}^{-1}$ (Fürst et al. 2010, and references therein). The distance to the system is $1.9 \pm 0.2 \mathrm{kpc}$ (Sadakane et al. 1985), making it one of the brightest persistent X-ray binaries in the sky, despite a mean luminosity of only $5 \times 10^{36} \mathrm{erg} \mathrm{s}^{-1}$ (Fürst et al. 2010). The neutron star seems to be significantly more massive than the canonical mass of $1.4 M_{\odot}$, with a lower limit of about $M_{\star}=1.8 M_{\odot}$ (van Kerkwijk et al. 1995; Quaintrell et al. 2003). Its flux is strongly variable, changing from barely detectable during off-states to up to $7 \mathrm{Crab}$ during giant flares (in the 20-40 keV energy band, Kreykenbohm et al. 2008; Staubert et al. 2004). This volatile behavior is a consequence of direct wind accretion, in which the material couples to the magnetic field before a stable accretion disk can form which then would mediate the accretion flow.
The wind of the super-giant is highly structured, or clumpy, which is a result of line-driven instability and the influence of the neutron star (see, among others, Owocki et al. 1988; Dessart \& Owocki 2005; Ducci et al. 2009; Fürst et al. 2010; Oskinova et al. 2012, and references therein). These clumps manifest themselves in two ways in the X-rays: as partially covering, variable absorption, and through a highly variable accretion rate. From the duration and brightness of flares, Fürst et al. (2010), using INTEGRAL data taken between 2003 and 2006, estimated that typical clumps have masses of $M \approx 5 \times 10^{19} \mathrm{~g}$ and radii of $r \approx 2 \times 10^{10} \mathrm{~cm}$, assuming a spherical clump geometry and homogeneous density. Similar values have also been found by Odaka et al. (2013) using Suzaku data and by Martínez-Núñez et al. (2013) using XMM-Newton data. The absorption column density of such clumps is around $2 \times 10^{22} \mathrm{~cm}^{-2}$, explaining naturally the $N_{\mathrm{H}}$ variations seen at early orbital phases by clumps moving through our line-of-sight (see, e.g., Haberl \& White 1990; Pan et al. 1994).

Cyclotron resonant scattering features (CRSFs, cyclotron lines for short) are an important feature of the X-ray spectra of accreting neutron stars. They are produced in strong magnetic fields, where electrons are quantized onto Landau-levels (Schönherr et al. 2007, and references therein). The energy of 
these levels is a strict function of the magnetic field strength. Photons with energies close to the Landau-levels are removed from the observed X-ray spectrum by scattering off these electrons. CRSFs appear as broad absorption lines due to Doppler broadening, and their energy is the only way to directly measure the magnetic field close to the surface of neutron stars. Vela X-1 shows a prominent harmonic line at $55 \mathrm{keV}$ and a weaker fundamental line at $25 \mathrm{keV}$ (Kendziorra et al. 1992; Kretschmar et al. 1996, 1997; Kreykenbohm et al. 2002; Maitra \& Paul 2013). The magnetic field strength can be calculated using

$$
B=\frac{E_{n}}{n \times 11.57 \mathrm{keV}} \times 10^{12} \mathrm{G},
$$

where $E_{n}$ is the energy of the fundamental $(n=1)$ or harmonic line $(n=2)$ line in $\mathrm{keV}$.

The detection of fundamental lines is complicated by the fact that in hard sources, with strong harmonic lines, their shape can be drastically altered to a point where the line is almost filled by spawned photons. Photon-spawning occurs when an electron remains in an excited Landau state after resonantly scattering, emitting another photon of similar energy when deexciting. These photons can escape the line-forming plasma, especially when their energy is at the wings of the fundamental line. Theoretical calculations by Schönherr et al. (2007) show that with increasing number of harmonic lines, the fundamental line gets shallower and can become undetectable in the observed $\mathrm{X}$-ray spectrum.

The centroid line energy of the CRSF is often found to vary with X-ray flux. This behavior was first observed with high significance in the transient source $\mathrm{V} 0332+53$, in which the line energy decreases with increasing flux (Tsygankov et al. 2010, 2006; Mowlavi et al. 2006). On the other hand, a positive correlation was found in Her X-1 (Staubert et al. 2007) and GX 304-1 (Yamamoto et al. 2011; Klochkov et al. 2012). In all these measurements, care has to be taken that the underlying continuum does not influence the line energy, as demonstrated for 4U 0115+634, where an anti-correlation was found using the NPEX continuum (Nakajima et al. 2006), but not when using a simpler cutoff-powerlaw (Müller et al. 2013).

Klochkov et al. (2011) performed a detailed study of the correlation between line energy and flux, confirming the behavior of Her X-1 and V $0332+53$ on shorter time-scales. They also found conclusive evidence that the power-law photon-index is changing with flux, and that it shows the opposite behavior to the line energy, i.e., that it decreases with flux in sources with a positive line-energy to flux correlation, and vice versa.

Both phenomena, the changing energy and the changing photon-index can be understood in terms of a change in altitude of the X-ray production region as function of flux, an idea put forward by Basko \& Sunyaev (1976) and discussed by Klochkov et al. (2011), Staubert et al. (2007), and Mowlavi et al. (2006), among others. With changing altitude, the local magnetic field changes, explaining the change in energy. At the same time, the local plasma temperature changes, which can result in a change in the observed photon-index. Depending on the overall luminosity, the correlation can switch from negative to positive, as described below, following the theory described in Becker et al. (2012).

At typical luminosities of Vela X-1 $\left(L_{\mathrm{x}} \leqslant 10^{37} \mathrm{erg} \mathrm{s}^{-1}\right)$, the matter is expected to be stopped very close to, or at, the stellar surface, so that the location of the line forming region is independent of the X-ray flux (Becker et al. 2012). At higher luminosities, a shock dominated by Coulomb interactions
Table 1

Observation Log

\begin{tabular}{lcccc}
\hline \hline ObsID & $\begin{array}{c}\text { Time Start } \\
\text { MJD (day) }\end{array}$ & $\begin{array}{c}\text { Time Stop } \\
\text { MJD (day) }\end{array}$ & $\begin{array}{c}\text { Exposure } \\
(\text { FPMA/B) }(\mathrm{ks})\end{array}$ & $\begin{array}{c}\text { Orbital } \\
\text { Phase }\end{array}$ \\
\hline 10002007 & 56117.6339 & 56117.9441 & $10.82 / 10.96$ & $0.655-0.690$ \\
30002007 & 56404.5275 & 56405.5559 & $41.67 / 41.92$ & $0.659-0.773$ \\
\hline
\end{tabular}

can form, stopping the matter above the stellar surface. The ram pressure of the in-falling material increases with higher accretion rate and pushes the shock region down into regions of higher magnetic field strength. This leads to a positive correlation of the line energy with flux and a hardening of the power-law component (as suggested by Staubert et al. 2007). If the local Eddington luminosity is exceeded, at the so-called critical luminosity, $L_{\text {crit }} \approx 3 \times 10^{37} \mathrm{erg} \mathrm{s}^{-1}$ for typical neutron star parameters, a radiation-dominated shock forms. The shock height is then positively correlated with the accretion rate as higher X-ray luminosity leads to a higher radiation pressure. The results is an observed an anti-correlation of the CRSF energy and correlation of the photon-index with flux.

The line behavior has not been investigated for Vela X-1, since no existing observations cover the necessary large range of luminosities with high spectral resolution. However, in a recent study using Suzaku, Odaka et al. (2013) found that the photon index $\Gamma$ is anti-correlated with the flux. This correlation indicates a sub-critical luminosity and would put Vela X-1 in the same group of sources as Her X-1 and GX 304-1. In the study by Odaka et al. (2013) the CRSF parameters were fixed in timeresolved spectroscopy and could not be studied as a function of luminosity.

In this paper we perform a spectral analysis on shorttime scales for two separate data-sets taken by the Nuclear Spectroscopic Telescope Array (NuSTAR; Harrison et al. 2013) about $1 \mathrm{yr}$ apart. In Section 2 we describe the data, their calibration and the software used. Section 3 discusses the lightcurve and variability of the hardness with time. Section 4 presents the analysis of the time-averaged spectrum. In Section 5 we perform time-resolved spectroscopy on pulse-to-pulse as well as on longer time-scales. In the Section 6 we discuss our results and give a physical interpretation of the parameters. In Section 7 we summarize our findings and give an outlook to future work.

\section{OBSERVATIONS AND DATA REDUCTION}

$N U S T A R$ consists of two independent gracing incidence telescopes, focusing X-rays between $3.0-79 \mathrm{keV}$ on corresponding focal planes consisting of cadmium zinc telluride (CZT) pixel detectors. NuSTAR provides unprecedented sensitivity and high spectral resolution at hard X-rays, ideally suited to study CRSFs in Vela X-1. The two focal planes are referred to as focal plane module (FPM) A and B.

NuSTAR observed Vela X-1 twice, once early in the mission as a calibration target for $\sim 11 \mathrm{ks}$ and later as a science target for $\sim 42 \mathrm{ks}$. We refer to these data-sets as observation I and II, respectively. Table 1 gives details about the observations, including the orbital phase, derived from the ephemeris by Kreykenbohm et al. (2008). While the orbital phase was very similar in both observations, Vela X-1 was a factor of about 3.5 brighter during observation I. Despite the shorter observation time of observation I, it provides similar signal-to-noise ratio $(\mathrm{S} / \mathrm{N})$ for the phase-averaged data due to the higher flux. 

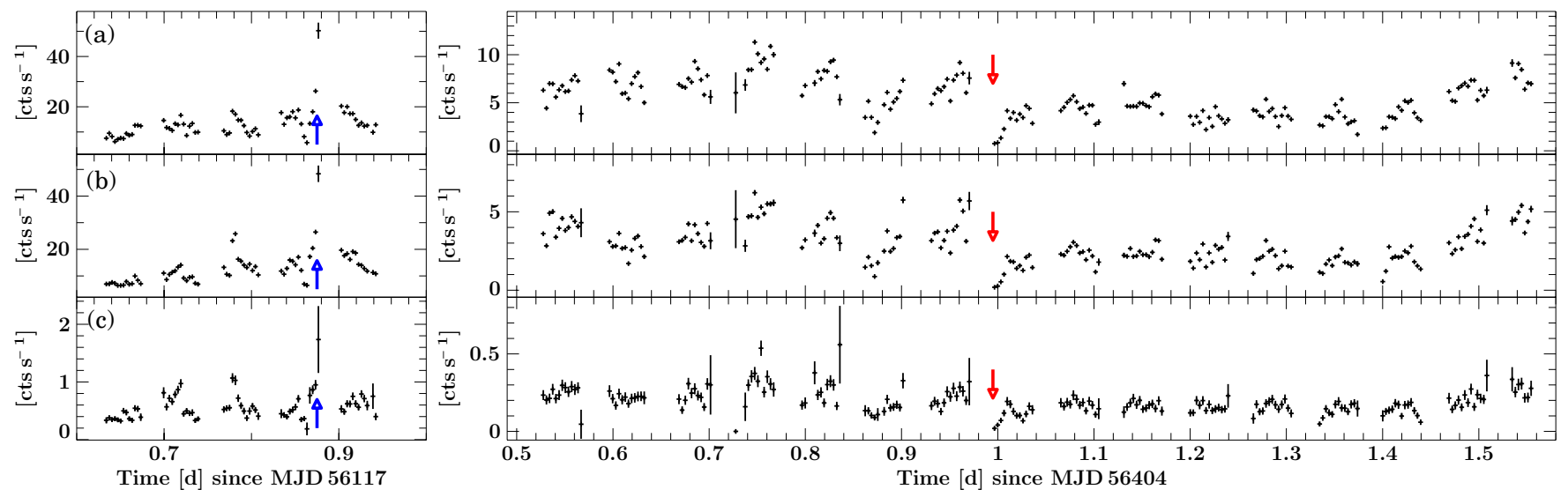

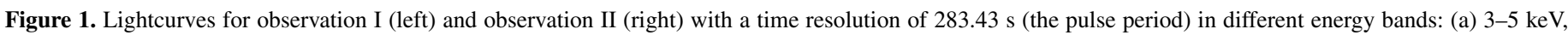

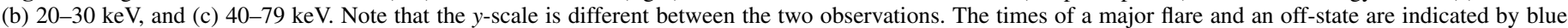
upward-pointing arrows and the red downward-pointing arrows, respectively.

(A color version of this figure is available in the online journal.)

We used the latest NUSTARDAS pipeline v1.2.0 and HEASOFT v6.13 to extract spectra and lightcurves. The exposure times given in Table 1 are the final exposure times after filtering for occultations, SAA passages and taking the detector dead-time into account. We barycentered the event times with the FTOOL barycen using the DE-200 solar system ephemeris and corrected for the binary orbit, using the ephemeris by Kreykenbohm et al. (2008). The data were analyzed using the Interactive Spectral Interpretation System (ISIS) v1.6.2 (Houck $\&$ Denicola 2000).

We extracted source spectra from a region with $150^{\prime \prime}$ radius around Vela X-1's FK5 coordinates, separately for FPMA and FPMB. As Vela X-1 is a very bright source, source photons illuminate the whole focal plane. To minimize their influence on the background estimation we extracted background spectra from a $80^{\prime \prime}$ radius region as far away from the source as possible. Since the background changes over the field-of-view, systematic uncertainties are formally introduced by this method. Vela X-1, however, is about a factor 5 brighter than the background even at the highest energies, such that the effect of residual uncertainties is negligible.

Both spectra were rebinned within ISIS to a minimal $\mathrm{S} / \mathrm{N}$ of $32,24,20,8$, and 2 adding at least $2,4,8,12$, and 18 channels for energies between 3.0-10, 10-28, 28-42, 42-54, and 54-79 keV, respectively. This rebinning allows us to use $\chi^{2}$ statistics for the fit. Uncertainties are given at the $90 \%$ confidence level $\left(\Delta \chi^{2}=2.7\right.$ for one parameter of interest), unless otherwise noted.

\section{LIGHTCURVES AND PULSE PROFILES}

The pulse-period of Vela X-1 varies in a random-walk like manner (de Kool \& Anzer 1993, and references therein), as is typical for a wind-accretor (Bildsten et al. 1997). We use the pulse period as the minimal integration time for our timeresolved spectroscopy, we therefore need to measure its value accurately. To do so, we used epoch-folding (Leahy 1987) on the 3.0-79 keV lightcurve of FPMA with $1 \mathrm{~s}$ time resolution. We find the period during observation II to be

$$
P=283.4290 \pm 0.0006 \mathrm{~s} .
$$

The uncertainty is estimated using a Monte Carlo simulation technique as described by Larsson (1996) with 2000 runs. The period is also consistent with the epoch folding results from observation I, with an uncertainty of $\Delta P=0.0024 \mathrm{~s}$.

Vela $\mathrm{X}-1$ is known to be highly variable on all time-scales, as is typical for a wind-accretor. Even within only a few pulseperiods, the flux can change by a factor of 5-7 (Kreykenbohm et al. 2008). This strong variability is also seen in the NuSTAR lightcurves (Figure 1). In observation I, a large flare was detected, indicated in Figure 1. Here, the dead-time corrected $3-79 \mathrm{keV}$ count-rate increased to $\approx 600$ counts $\mathrm{s}^{-1}$, from an average of $\approx 150$ counts $\mathrm{s}^{-1}$ during the whole observation. Observation II was fainter overall with an average 3-79 keV count-rate of only $\approx 48$ counts $\mathrm{s}^{-1}$ and it did not show huge flares. Instead, an off-state was detected, highlighted in Figure 1. In this off-state, the count-rate dropped to $3-4$ counts s$^{-1}$.

Flux variability is often associated with a change in the spectrum as well. In Figure 2 we show the hardness-ratios HR, calculated as $\mathrm{HR}=(H-S) /(H+S)$, between three energy bands (a low-energy ratio in the top panel, and a high-energy ratio in the bottom panel). The flare in observation I does not seem to be associated with a strong spectral change, especially for the ratio between $S=3-5 \mathrm{keV}$ and $H=20-30 \mathrm{keV}$. This behavior is similar to many of the flares studied by Staubert et al. (2004), Kreykenbohm et al. (2008) and Odaka et al. (2013).

During the off-state in observation II, strong changes in both hardness ratios are evident, with the spectrum becoming softer in the low-energy ratio and harder in the high-energy ratio, i.e., the spectrum becomes flatter overall. A detailed analysis of offstates in Suzaku data is presented by Doroshenko et al. (2011), confirming this overall behavior.

The strong changes of both flux and hardness necessitate an analysis of the spectral variability on short time-scales. We therefore extracted one spectrum per pulse. However, the $\mathrm{S} / \mathrm{N}$ in each individual spectrum is not high enough to fit all parameters in a typical absorbed power-law model with a high-energy cutoff. We therefore first fit the time-averaged spectra of both observations separately in Section 4 and use these parameters for the pulse-to-pulse fits in Section 5.

\section{TIME-AVERAGED SPECTROSCOPY}

To model the time-averaged spectrum, we use a power-law continuum with a Fermi-Dirac cut-off (FDcut; Tanaka 1986). This model is often applied to Vela X-1 (see, e.g., Kreykenbohm et al. 2008). A simple cutoffpl does not fit the data, and the 

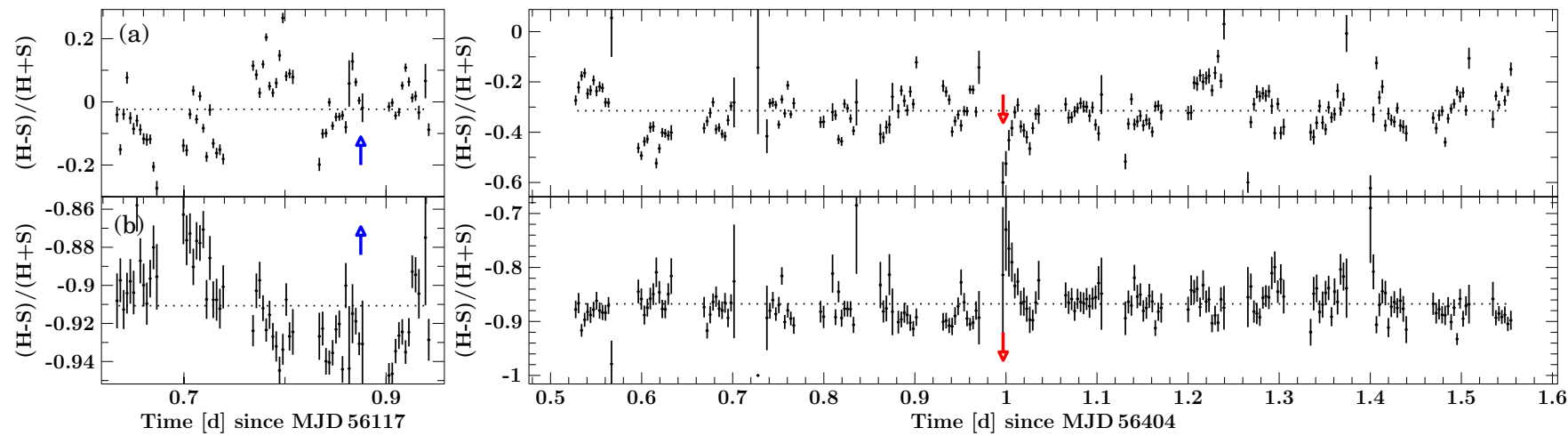

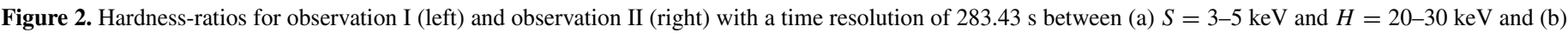

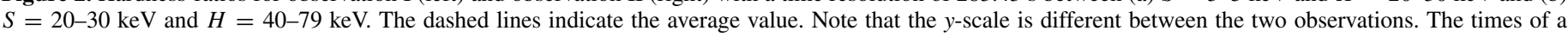
major flare and an off-state are indicated by blue upward-pointing arrows and the red downward-pointing arrows, respectively.

(A color version of this figure is available in the online journal.)

NPEX model (Makishima et al. 1999) can result in unrealistic CRSF line parameters (Müller et al. 2013). To confirm our results, we also applied the NPEX model, as described in Section 5. The FDcut-model is characterized by the photon index $\Gamma$, the cut-off energy $E_{\text {cut }}$ and the folding energy $E_{\text {fold }}$ in the following form:

$$
F(E) \propto E^{-\Gamma} \times\left(1+\exp \left(\frac{E-E_{\text {cut }}}{E_{\text {fold }}}\right)\right)^{-1} .
$$

Even though the two telescopes of $N U S T A R$ are almost identical, small differences in the measured flux are possible, with the difference being below 5\%. To allow for this crosscalibration difference, we include a constant in the model, $C_{\text {FPMB }}$, which gives the relative normalization of FPMB with respect to FPMA.

The neutron star is embedded deeply in the stellar wind and an intrinsic absorption column of the order of $10^{23} \mathrm{~cm}^{-2}$ is often observed at the late orbital phases where both $N U S T A R$ observations took place $(\sim 0.6$; see Table 1$)$. We describe this absorption column using an updated version of the tbabs model (Wilms et al. 2000) and the corresponding abundances and cross-sections (Verner et al. 1996). The absorbing material is not smooth, but structured or clumpy (see, e.g., Fürst et al. 2010). To take this structure into account, we use a partial covering model with two absorption columns, $N_{\mathrm{H}, 1}$ and $N_{\mathrm{H}, 2}$, and a covering fraction $0<\mathrm{CF}<1$.

Vela X-1 also shows fluorescence lines in its soft X-ray spectrum, associated with $\mathrm{FeK} \alpha$ and $\mathrm{FeK} \beta$. Their energies are usually close to that of neutral iron (Watanabe et al. 2006). We modeled these lines with two independent Gaussians around $6.4 \mathrm{keV}$ and $7.1 \mathrm{keV}$. For neutral iron we would expect that the flux of $\mathrm{FeK} \beta$ is about 0.13 the flux $\mathrm{FeK} \alpha$ (Palmeri et al. 2003). However, because the overlapping Fe K-edge at $7.1 \mathrm{keV}$, the FeK $\beta$ flux is difficult to constrain with $N u S T A R$ and can be artificially higher than expected from theoretical calculations.

To describe the cyclotron lines we use a multiplicative absorption line model, $\operatorname{CRSF}(E)=\exp (-\tau(E))$, with a Gaussian optical depth profile (gabs in XSPEC):

$$
\tau(E)=\frac{d}{\sigma \sqrt{2 \pi}} \exp \left(-\frac{1}{2}\left(\frac{E-E_{\mathrm{cyc}}}{\sigma}\right)^{2}\right),
$$

with line depth $d$ and width $\sigma$. We use the subscripts "CRSF,F" and "CRSF,H" to denote parameters for the fundamental and the harmonic line, respectively.
When we apply this model without allowing for a fundamental line, the overall continuum shape is well described, but the obtained $\chi^{2}$ values are unacceptable $\left(\chi^{2} /\right.$ dof $=791.4 / 559$ for observation I and 897.1/553 for observation II). Adding the fundamental line does results in almost the same quality of fit for observation I but improves it dramatically for observation II $\left(\chi^{2} /\right.$ dof $=789.7 / 557$ and $614.2 / 551$, respectively). Since the fundamental line is relatively weak, and its width unconstrained, we required its width $\sigma_{\mathrm{CRSF}, \mathrm{F}}$ to be half the width of the harmonic line, $\sigma_{\mathrm{CRSF}, \mathrm{H}}$ in both observations, as indicated by the best-fit of observation II. A narrow fundamental line prevents it from becoming so strong as to unphysically influence the other model parameters. Kreykenbohm et al. (2002) also found that the fundamental line is consistently narrower in pulse-resolved analysis.

Residuals remain around $10 \mathrm{keV}$, which can be very well described with a broad Gaussian absorption line. Its physical origin is unexplained, but it has been observed in Vela X-1 before (e.g., La Barbera et al. 2003), as well as in many other CRSF sources (Coburn et al. 2002), always at $\sim 10 \mathrm{keV}$. The current version of the NuSTAR responses show higher uncertainties around the tungsten edge at $\sim 10 \mathrm{keV}$. These response features are, however, clearly weaker and have a different shape than the residuals described here (Fürst et al. 2013). The $10 \mathrm{keV}$ feature is more prominent in observation I and the final fit gives values of $\chi^{2} /$ dof $=587.5 / 554$ and $570.8 / 548$ for the two observations, respectively.

This final model can be summarized as

$$
\begin{aligned}
I(E)= & \left(\mathrm{CF} \times N_{\mathrm{H}, 1}+(1-\mathrm{CF}) \times N_{\mathrm{H}, 2}\right) \\
& \times(\mathrm{FDcut} \times \mathrm{CRSF}(\mathrm{F}) \times \mathrm{CRSF}(\mathrm{H}) \\
& +10 \mathrm{keV}-\text { line }+\mathrm{FeK} \alpha+\mathrm{FeK} \beta) .
\end{aligned}
$$

The data and best-fit model for both observations are shown in Figure 3. In the residual panels $b$ and $c$ in both figures the fundamental line and the $10 \mathrm{keV}$ feature are turned off, respectively, to show their contribution to the overall continuum shape. It is clear that both features are variable, and that the fundamental line is almost invisible in observation I. Table 2 lists the best-fit parameters for both observations. Besides the variability of the cyclotron lines, the photon index $\Gamma$ also changes significantly between the two observations, while the cutoff and folding energy are almost constant. The second absorber $N_{\mathrm{H}, 2}$ could not be constrained in observation II, so we fixed it to 0 . 

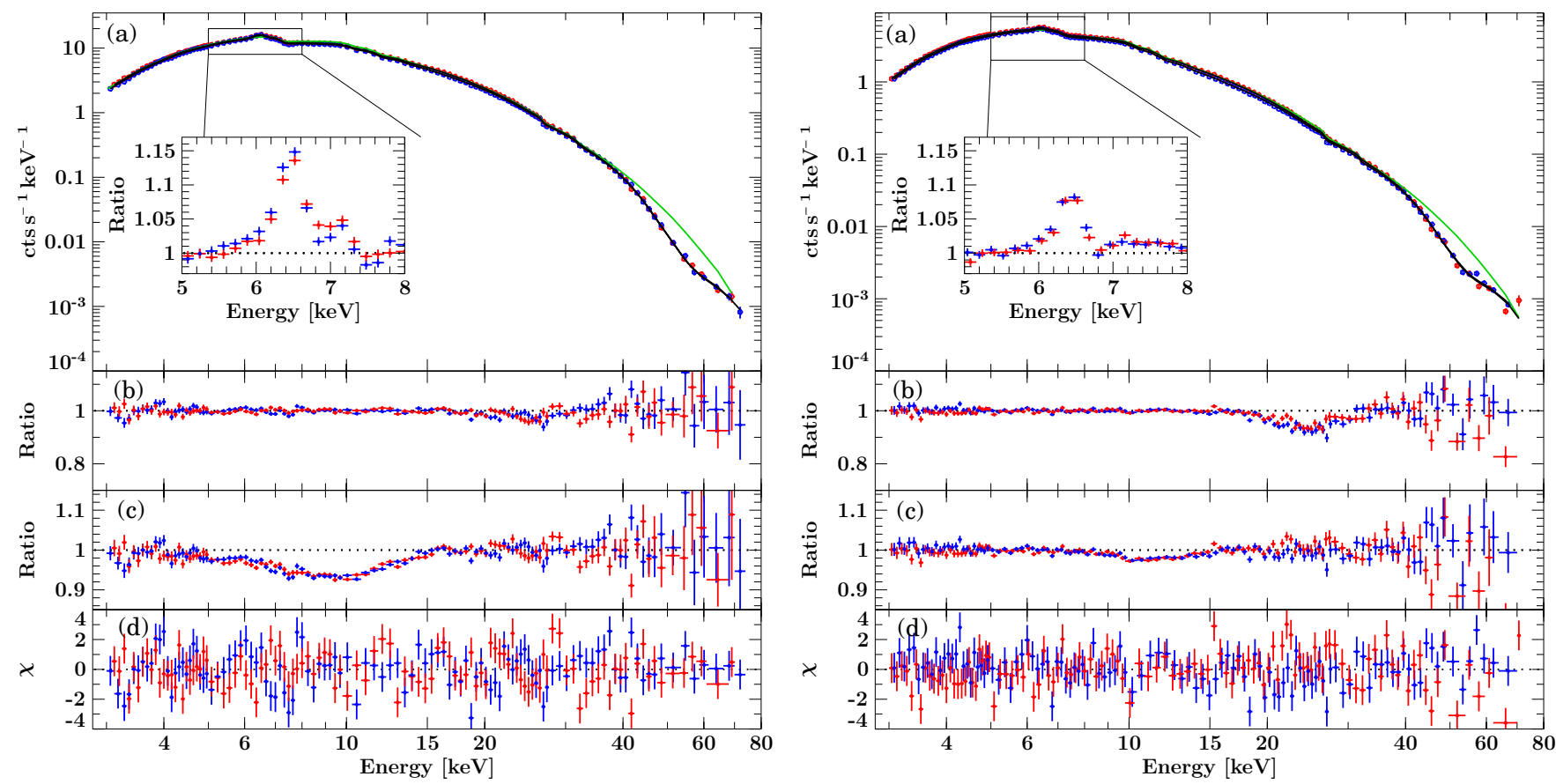

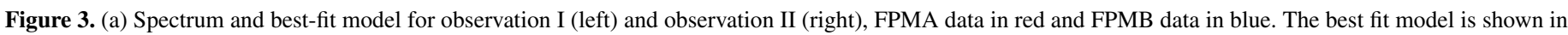

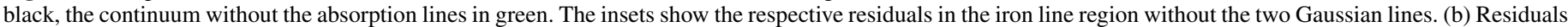
without the fundamental CRSF line. (c) Residuals without the $10 \mathrm{keV}$-feature. (d) Residuals to the best-fit in units of $\sigma$.

(A color version of this figure is available in the online journal.)

Table 2

Fit Parameters for the Best-fit Time-averaged Model

\begin{tabular}{|c|c|c|}
\hline Parameter & Obs. I & Obs. II \\
\hline $\mathrm{CF}$ & $0.823_{-0.053}^{+0.022}$ & $0.868 \pm 0.007$ \\
\hline$N_{\mathrm{H}, 1}\left(10^{22} \mathrm{~cm}^{-2}\right)$ & $34.2_{-2.1}^{+5.7}$ & $26.0_{-1.0}^{+0.9}$ \\
\hline$N_{\mathrm{H}, 2}\left(10^{22} \mathrm{~cm}^{-2}\right)$ & $0.9_{-0.9}^{+4.2}$ & $\ldots$ \\
\hline$\Gamma$ & $0.88_{-0.06}^{+0.05}$ & $1.28_{-0.05}^{+0.04}$ \\
\hline$E_{\text {cut }}(\mathrm{keV})$ & $19.3_{-3.8}^{+2.5}$ & $22.3_{-3.9}^{+2.8}$ \\
\hline$E_{\text {fold }}(\mathrm{keV})$ & $10.5_{-0.5}^{+0.9}$ & $13.3_{-0.8}^{+1.0}$ \\
\hline$E_{\mathrm{CRSF}, \mathrm{H}}(\mathrm{keV})$ & $55.4_{-1.0}^{+1.3}$ & $53.2_{-0.8}^{+0.9}$ \\
\hline$\sigma_{\mathrm{CRSF}, \mathrm{H}}(\mathrm{keV})$ & $8.0_{-1.0}^{+1.3}$ & $6.8_{-0.7}^{+0.9}$ \\
\hline$d_{\mathrm{CSRF}, \mathrm{H}}(\mathrm{keV})$ & $20_{-5}^{+8}$ & $14.0_{-2.3}^{+3.2}$ \\
\hline$E_{\mathrm{CRSF}, \mathrm{F}}(\mathrm{keV})$ & $25.1_{-2.4}^{+2.0}$ & $24.8 \pm 0.6$ \\
\hline$d_{\mathrm{CSRF}, \mathrm{F}}(\mathrm{keV})$ & $0.24_{-0.13}^{+0.20}$ & $0.61_{-0.12}^{+0.18}$ \\
\hline$A(\mathrm{Fe} \mathrm{K} \alpha)^{\mathrm{a}}$ & $0.038_{-0.019}^{+0.263}$ & $\left(0.93_{-0.08}^{+0.09}\right) \times 10^{-3}$ \\
\hline$E(\mathrm{Fe} \mathrm{K} \alpha)(\mathrm{keV})$ & $6.474 \pm 0.010$ & $6.4393_{-0.0393}^{+0.0008}$ \\
\hline$\sigma(\mathrm{Fe} \mathrm{K} \alpha)(\mathrm{keV})$ & $0.065_{-0.065}^{+0.030}$ & $\leqslant 0.07$ \\
\hline$A(\mathrm{Fe} \mathrm{K} \beta)^{\mathrm{a}}$ & $0.008_{-0.008}^{+0.068}$ & $\left(3.3_{-1.0}^{+1.3}\right) \times 10^{-4}$ \\
\hline$E(\mathrm{Fe} \mathrm{K} \beta)(\mathrm{keV})$ & $7.084_{-0.008}^{+0.077}$ & $7.39 \pm 0.12$ \\
\hline$\sigma(\mathrm{Fe} \mathrm{K} \beta)(\mathrm{keV})$ & $0.00022_{-0.00022}^{+0.07167}$ & $0.30_{-0.12}^{+0.16}$ \\
\hline$A(10 \mathrm{keV})$ & $-0.19_{-1.82}^{+0.14}$ & $\left(-1.3_{-0.9}^{+0.5}\right) \times 10^{-3}$ \\
\hline$E(10 \mathrm{keV})(\mathrm{keV})$ & $8.6_{-0.7}^{+0.5}$ & $10.9 \pm 0.4$ \\
\hline$\sigma(10 \mathrm{keV})(\mathrm{keV})$ & $2.8_{-0.5}^{+0.6}$ & $1.9_{-0.5}^{+0.6}$ \\
\hline $\mathcal{F}_{3-79 \mathrm{keV}^{\mathrm{b}}}$ & $11.22_{-0.08}^{+0.10}$ & $3.206 \pm 0.016$ \\
\hline$C_{\text {FPMB }}$ & $1.0491 \pm 0.0020$ & $1.0259 \pm 0.0018$ \\
\hline$\chi^{2} /$ dof & $616.63 / 565$ & $589.12 / 560$ \\
\hline$\chi_{\mathrm{red}}^{2}$ & 1.091 & 1.052 \\
\hline
\end{tabular}

Notes.

${ }^{\text {a }}$ In $\mathrm{ph} \mathrm{s}^{-1} \mathrm{~cm}^{-2}$.

${ }^{\mathrm{b}}$ In $\mathrm{keV} \mathrm{s}^{-1} \mathrm{~cm}^{-2}$.

\section{TIME-RESOLVED SPECTROSCOPY}

Having established a good description of the time-averaged spectrum, we use this model to study the variability of the spectral parameters on a pulse-to-pulse basis. Thanks to the high sensitivity of $N U S T A R$, it is possible to obtain a high quality spectrum for each individual pulse, i.e., rotation of the neutron star. Higher time resolution is not sensible, as the spectrum changes strongly with pulse phase (see, e.g., Kreykenbohm et al. 2002; La Barbera et al. 2003; Maitra \& Paul 2013). This approach is similar to the analysis of Fürst et al. (2011) for GX 301-2 using XMM-Newton, but thanks to NuSTAR's broad band coverage changes in the continuum and the cyclotron line can also be studied.

We applied the time-averaged model, but due to the reduced $\mathrm{S} / \mathrm{N}$ in the pulse-to-pulse spectra we had to fix some parameters to their respective time-averaged values. We only let the covering fraction $\mathrm{CF}$, one absorption column $N_{\mathrm{H}, 1}$, the photon index $\Gamma$, and the normalization of all lines or line-like features (fundamental CRSF, harmonic CRSF, $10 \mathrm{keV}$ feature, as well as FeK $\alpha$ and $\mathrm{FeK} \beta$ ) vary. We chose the photon index $\Gamma$ as the variable continuum parameter, as it shows the strongest variation between observation I and II. With this approach we obtained very good $\chi_{\text {red }}^{2}$ values for all spectra, with an average $\chi_{\text {red }}^{2} \approx 1.05$. For the observation I fits, $N_{\mathrm{H}, 2}$ was fixed to $9 \times 10^{21} \mathrm{~cm}^{-2}$, while for observation II it was set to 0 , the respective values of the time-averaged models.

Figure 4 show the measured variation of the model parameters as a function of time. All parameters show variability with time, most prominently $N_{\mathrm{H}, 1}, \Gamma$, and CF. However, the typical timescale for significant changes seems to be longer than single pulses. We use the fact that consecutive data often show similar spectral parameters to add them up in order to obtain better $\mathrm{S} / \mathrm{N}$. During observation I, we added half of each $\sim 90 \mathrm{~min}$ 


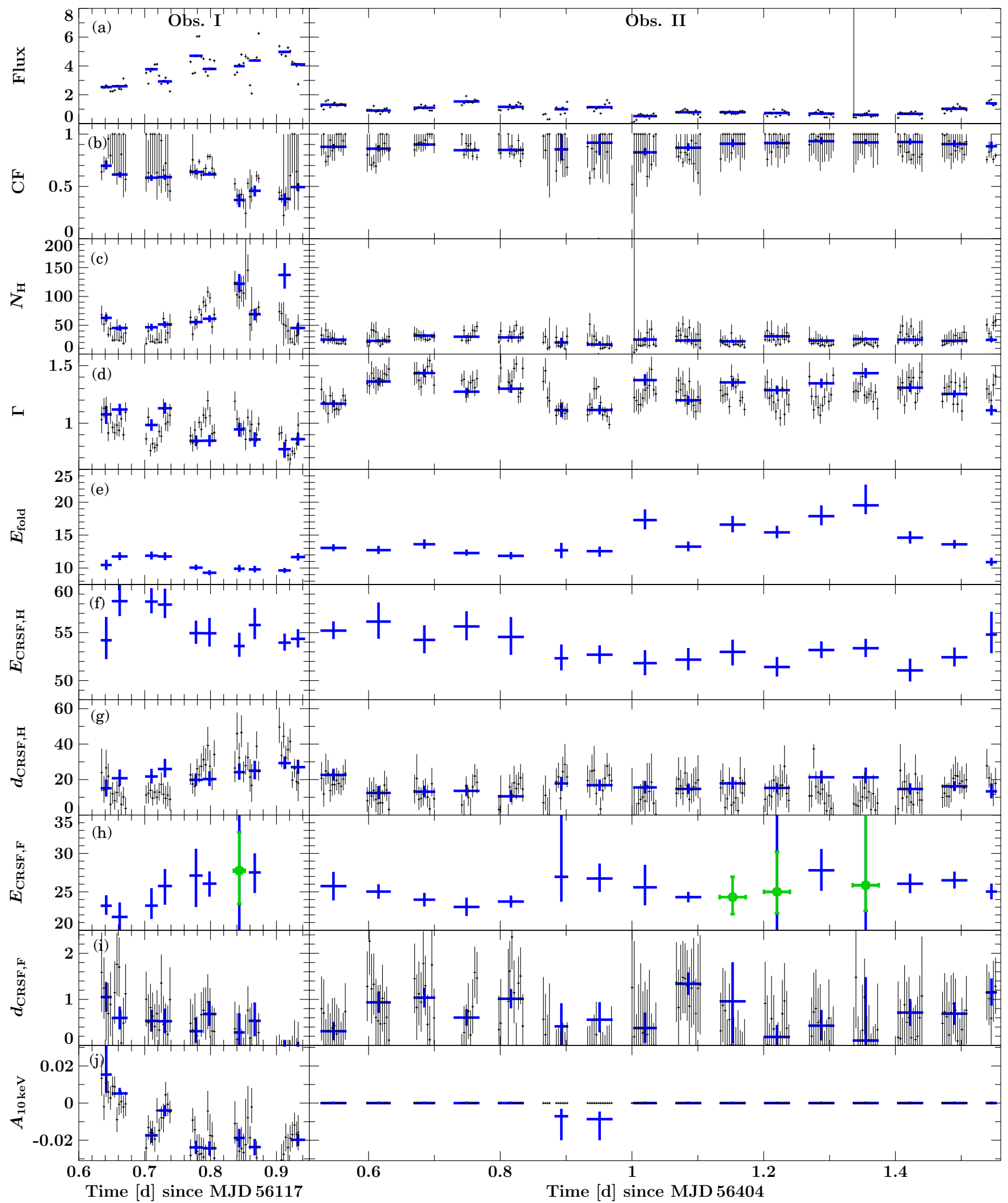

Figure 4. Results of the pulse-to-pulse (black) and ks-integrated (blue) spectral fits for both observations (observation I in the left column, observation II in the right column). (a) Unabsorbed flux in the 20-40 keV energy band in $\mathrm{keV} \mathrm{s}^{-1} \mathrm{~cm}^{-2}$, (b) covering fraction, (c) absorption column in $10^{22} \mathrm{~cm}^{-2}$, (d) photon index $\Gamma$, (e) folding energy $E_{\text {fold }}$ in $\mathrm{keV}$, (f) centroid energy of the harmonic CRSF in keV; (g) depth of the harmonic CRSF in keV; (h) centroid energy of the fundamental CRSF in $\mathrm{keV}$, green values are results with the depth frozen to its best fit value, see text for details; (i) depth of the fundamental CRSF in keV; and (j) normalization of the $10 \mathrm{keV}$-feature in $\mathrm{ph} \mathrm{s}^{-1} \mathrm{~cm}^{-2}$.

(A color version of this figure is available in the online journal.) 
Table 3

Fit Parameters for the Off-state

\begin{tabular}{lcc}
\hline \hline Parameter & FDcut & Powerlaw \\
\hline $\mathrm{CF}$ & $1.00_{-0.21}^{+0.00}$ & $1.00_{-0.17}^{+0.00}$ \\
$N_{\mathrm{H}, 1}\left(10^{22} \mathrm{~cm}^{-2}\right)$ & $11.6_{-3.0}^{+6.8}$ & $17_{-4}^{+12}$ \\
$\Gamma$ & $1.52 \pm 0.10$ & $1.91_{-0.10}^{+0.14}$ \\
$d_{\mathrm{CSRF}, \mathrm{H}}(\mathrm{keV})$ & $\leqslant 17$ & $20_{-17}^{+20}$ \\
$d_{\mathrm{CSRF}, \mathrm{F}}(\mathrm{keV})$ & $\leqslant 0.9$ & $\leqslant 1.7$ \\
$A(\mathrm{Fe} \mathrm{K} \alpha)^{\mathrm{a}}$ & $\left(0.9_{-0.8}^{+1.0}\right) \times 10^{-3}$ & $\left(1.5_{-1.5}^{+2.3}\right) \times 10^{-4}$ \\
$A(\mathrm{Fe} \mathrm{K} \beta)^{\mathrm{a}}$ & $\leqslant 5 \times 10^{-4}$ & $\leqslant 1.2 \times 10^{-4}$ \\
$\mathcal{F}_{3-79 \mathrm{keV}}$ & $0.256 \pm 0.010$ & $0.319_{-0.022}^{+0.025}$ \\
$\chi^{2} / \mathrm{dof}$ & $122.82 / 90$ & $104.22 / 90$ \\
$\chi_{\text {red }}^{2}$ & 1.365 & 1.158 \\
\hline
\end{tabular}

Notes.

a In $\mathrm{ph} \mathrm{s}^{-1} \mathrm{~cm}^{-2}$.

b In $\mathrm{keV} \mathrm{s}^{-1} \mathrm{~cm}^{-2}$.

satellite orbit, yielding $\sim 1.1 \mathrm{ks}$ integration time per spectrum. We removed the high $N_{\mathrm{H}}$ data points around MJD 56117.85. Observation II was less bright, so we integrated mostly over one satellite orbit, i.e., about $2.4 \mathrm{ks}$, cutting out the dip around MJD 56405.0 and the phase of dramatic change in $\Gamma$ at MJD 56404.88 .

The improved $\mathrm{S} / \mathrm{N}$ allows us to additionally let the folding energy $E_{\text {fold }}$, the energies of the two cyclotron lines, as well as energy of the FeK $\alpha$ line vary. The results are shown in blue in Figure 4, and follow the pulse-to-pulse results overall very well. Some ks-integrated results show significant deviations from the corresponding pulse-to-pulse results. These deviations originate in part from changes in parameters like $E_{\text {fold }}$ and $E_{\mathrm{CRSF}, \mathrm{H}}$ which were frozen in the pulse-to-pulse analysis, but were allowed to vary in the ks-integrated data. Additionally, by summing over strong spectral changes on a pulse-to-pulse basis, the resulting ks-integrated spectrum might have an slightly different shape, resulting in a shifted best fit. These deviations, however, do not influence our conclusions.

The covering fraction $\mathrm{CF}$ is almost always larger than 0.8 and often consistent with 1 , albeit with relatively large error bars in the pulse-to-pulse results. However, around MJD 56117.85 in observation I, the covering fraction drops significantly below 0.5 , while at the same time $N_{\mathrm{H}}$ rises to very high values, on the order of $10^{24} \mathrm{~cm}^{-2}$. The photon index varies by about $0.3-0.4$ in each observation, and is, as expected from the phase-averaged results, clearly different between observation I and II.

Significant variations in the energy and depth of the harmonic CRSF are clearly visible in both observations (Figures 4(f) and $(\mathrm{g})$ ). The fundamental line strength changes from a clear detection in a single pulse to being below the detection limit, even in the ks-integrated data. In the last orbit of observation I the best-fit requires a strength $d_{\mathrm{CRSF}, \mathrm{F}}$ of 0 , resulting in an unconstrained energy. We therefore fixed the line energy to half the energy of the harmonic line in order to estimate an upper limit of the line depth at this energy. In four other orbits, the line depth was consistent with 0 (90\% confidence), also leading to an unconstrained line energy. In these cases, we fix the strength to the best-fit value, and recalculate the energy confidence interval, shown in green in Figure 4(h). This is the first time that the evolution of the cyclotron lines in Vela X-1 has been studied on these short time-scales.

The $10 \mathrm{keV}$ feature is most prominent at the beginning of observation I. Observation II almost never requires this feature

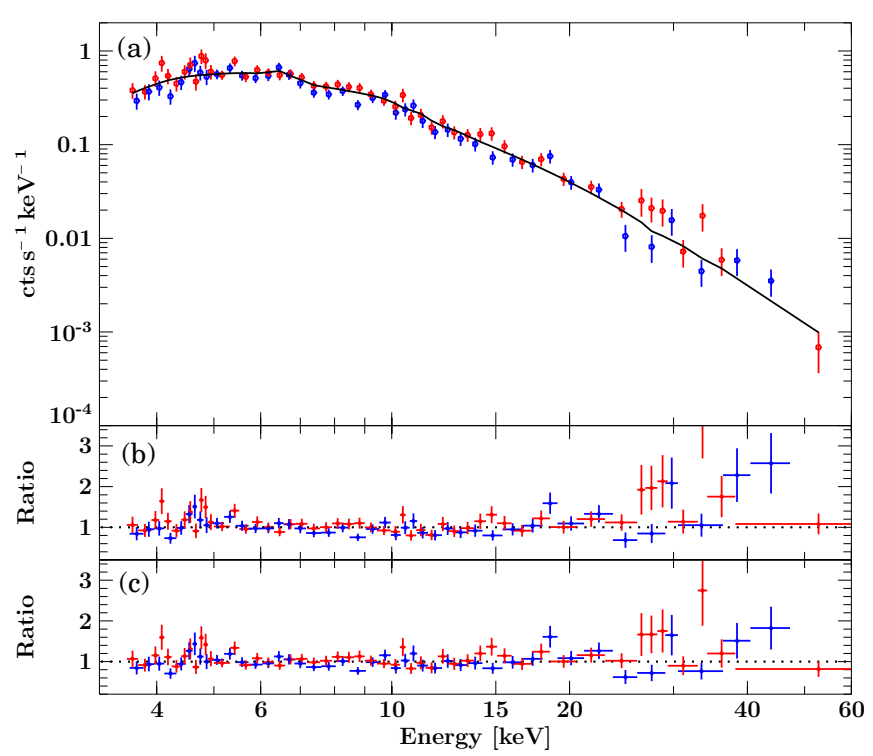

Figure 5. (a) Spectrum of the off-state with the best-fit power-law model in black, FPMA data in red and FPMB data in blue. (b) Residuals with the standard pulse-to-pulse FDcut model (c) Residuals with power-law only continuum.

(A color version of this figure is available in the online journal.)

for a good fit, either in pulse-to-pulse or in ks-integrated data. Only two spectra around MJD 56404.9 and MJD 56404.95 required a small but significant contribution from this feature. The energy of the $10 \mathrm{keV}$-feature (not shown) is, when detected, in all cases very close to $10 \mathrm{keV}$ and does not show significant variation with time.

To assess if the strong variability of the spectral parameters is independent of the chosen continuum, we also describe the ks-integrated spectra with the NPEX model. We obtain fits of similar quality in terms of $\chi_{\text {red }}^{2}$. The parameters comparable between the models, i.e., the CRSF, the absorption and the iron line parameters are all consistent within the uncertainties and follow a similar evolution with time. We therefore rule out a systematic effect of the chosen continuum on the important spectral parameters.

\subsection{The Spectrum of the Off-state}

While the bright flare in observation I did not show any significant spectral changes, the off-state in observation II does (see Figure 2). We therefore extracted a spectrum covering only the off-state, which lasted for $450 \mathrm{~s}$ before the hardness-ratio levels out and clear pulsations are visible in the lightcurve again (Figure 5). The statistics of this spectrum are comparable to the pulse-to-pulse spectra, but applying the same model gave a high value of $\chi^{2}=122.8$ for 90 dof, see Table 3 .

There is no evidence for a roll-over at higher energies, so we remove the FDcut component completely and describe the continuum with a power-law only (see Figure 5(c)). The best fit gives an acceptable $\chi^{2}=104.2$ with also 90 dof. The powerlaw index increases to a very high value, see the second column of Table 3. The absorption column density, however, does not rise to particularly high values, so the off-state is clearly not due to an absorption event.

The cyclotron lines are not detected significantly in the off-state spectrum. The depth of the harmonic line is highly uncertain due to the lack of signal above $50 \mathrm{keV}$. The overall spectral shape is very similar to the one found by Doroshenko 
et al. (2011) in Suzaku data, though the NuSTAR data provide a significantly higher $\mathrm{S} / \mathrm{N}$ above $30 \mathrm{keV}$.

\section{DISCUSSION}

\subsection{Flux Dependence of the Cyclotron Lines}

Using the results from the ks-integrated spectra, we plot the energies of the CRSFs as function of luminosity in Figure 6. A clear increase of the energy of the harmonic CRSF with luminosity is visible, which has not significantly been seen in a persistent high-mass X-ray binary (HMXB) before. The evolution of the energy of the fundamental line with luminosity is difficult to interpret and influenced by the fact that the width is poorly constrained. Its energy may also be influenced by photon-spawning, as detailed in Section 6.2.

To put the data into context, a sample of other sources and their CRSF-luminosity relation, as well as $L_{\text {coul }}$ and $L_{\text {crit }}$ are also shown in Figure 6, following Figure 2 of Becker et al. (2012). The luminosity at which Coulomb interactions start to dominate, $L_{\text {coul }}$, and the critical luminosity, where the local Eddington limit is exceeded, $L_{\text {crit }}$, are calculated under the same assumptions as in Becker et al. (2012). Additionally we show both luminosities for a neutron star with a higher mass of $M_{\star}=1.8 M_{\odot}$, the lower mass limit of Vela X-1.

Recently Hemphill et al. (2013), using INTEGRAL data as well as values from the literature, showed that 4U 1907+09 shows an indication for a correlation between line energy and luminosity at luminosities around $2 \times 10^{36} \mathrm{erg} \mathrm{s}^{-1}$. However, the correlation is strongly dependent on one measurement at high luminosities by Rivers et al. (2010) and has systematic uncertainties due to comparing values from the literature measured with different continua and are therefore not shown in Figure 6.

From Figure 6 it seems clear that Vela X-1 is situated in the regime where the in-falling matter is only stopped at the stellar surface, and no correlation between luminosity and line energy is expected. A $0535+26$ seems to be in the same regime and follows this predicted behavior. Nonetheless the energy of the harmonic CRSF of Vela X-1 shows a clear correlation with luminosity. These data are the first clear evidence for a correlation below $L_{\mathrm{x}} \sim 10^{36} \mathrm{erg} \mathrm{s}^{-1}$. In the following, we discuss a possible explanation for the clear correlation found in Vela X-1 at these low luminosities.

To calculate $L_{\text {coul }}$, at which the Coulomb interaction will stop the in-falling matter above the stellar surface in a shock, Becker et al. (2012) first calculate the radius of the accretion column in their Equation (23) with

$$
\begin{aligned}
r= & 1.93 \times\left(\frac{\Lambda}{0.1}\right)^{-\frac{1}{2}}\left(\frac{M_{\star}}{1.4 M_{\odot}}\right)^{-\frac{1}{14}}\left(\frac{R_{\star}}{10 \mathrm{~km}}\right)^{\frac{11}{14}} \\
& \times\left(\frac{B_{\star}}{10^{12} G}\right)^{-\frac{2}{7}}\left(\frac{L_{x}}{10^{37} \mathrm{erg} \mathrm{s}^{-1}}\right)^{-\frac{1}{7}}
\end{aligned}
$$

where $M_{\star}, R_{\star}$, and $B_{\star}$, are the mass, radius and surface magnetic field of the neutron star, respectively. The parameter $\Lambda$ is defined by Lamb et al. (1973): $\Lambda=1$ for spherical accretion and $\Lambda<1$ for disk accretion. That is, $\Lambda$ summarizes the interaction between the magnetic field and the surrounding medium, describing the Alfvén radius. It is the least well known variable in the equation and a likely way to explain why Vela $X-1$ is in a different accretion regime despite its low luminosity.

Vela X-1 accretes directly from the stellar wind, a scenario best described by $\Lambda=1$. Becker et al. (2012) assume a stable

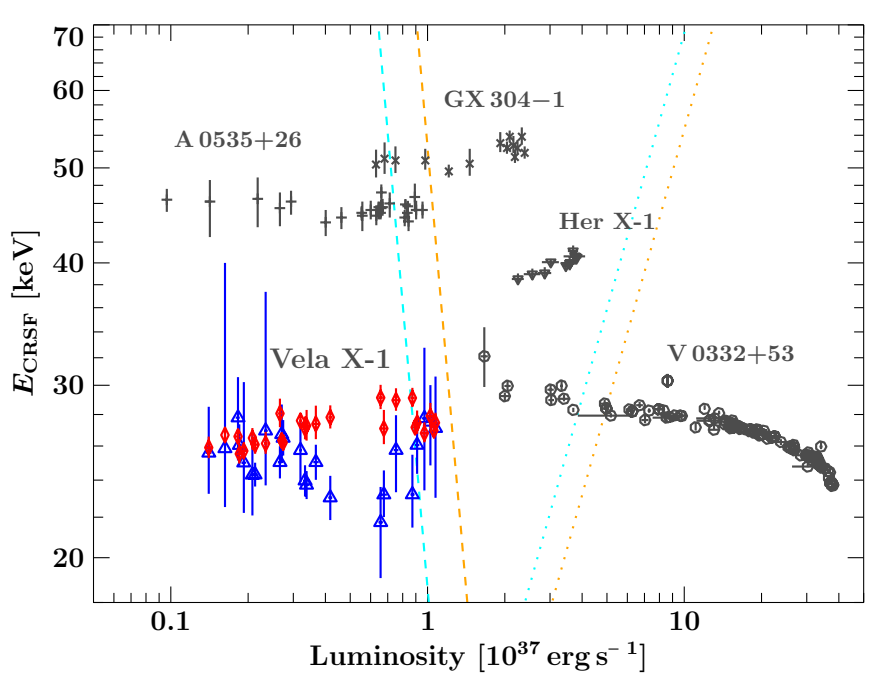

Figure 6. Fundamental cyclotron line energy as function of luminosity for different sources. The energy of the fundamental line of Vela X-1 is shown in blue using upright triangles, the harmonic line divided by a factor of two in red diamonds. The dashed lines indicate the Coulomb breaking limit, $L_{\mathrm{Cou}}$ and the dotted lines the critical luminosity, $L_{\text {crit }}$, following Becker et al. (2012) and using $\Lambda=0.1$. The left-hand line (cyan) of each pair assumes $M_{\star}=1.4 M_{\odot}$, the right-hand line (golden) $M_{\star}=1.8 M_{\odot}$. A 0535+26 data (crosses) are obtained from Caballero et al. (2007), V 0332+53 (circles) are from Tsygankov et al. (2010), Her X-1 (down-pointing triangles) are from Staubert et al. (2007), and GX 304-1 (asterisks) are from Yamamoto et al. (2011).

(A color version of this figure is available in the online journal.)

accretion disk and set $\Lambda=0.1$ in their calculation. Additionally $M_{\star} \geqslant 1.8 M_{\odot}$ for Vela X-1. With these values, we obtain $r \approx 0.4 \mathrm{~km}$ for the observed luminosities of Vela X-1 in the spherical accretion case. This is a much smaller radius compared to $r \approx 1.3 \mathrm{~km}$ for the standard disk accretion case.

The high pulsed fraction of Vela X-1 provides observational evidence for a very small accretion radius. If the $\mathrm{X}$-rays are produced in a very localized region, the effects of the rotation of the neutron star will be more pronounced. Calculations taking relativistic light-bending into account are required to constrain the accretion column radius, but are beyond the scope of this paper.

The drastically reduced radius of the accretion column directly leads to a reduced Coulomb luminosity. Assuming $B_{\star}=$ $2.59 \times 10^{12} \mathrm{G}$, i.e., a surface fundamental cyclotron energy of $E_{\star}=30 \mathrm{keV}$, we compute $L_{\text {coul }}=3.34 \times 10^{36} \mathrm{erg} \mathrm{s}^{-1}$ using Equation (54) of Becker et al. (2012). This luminosity is indicated by the vertical dashed line in Figure 7(a), moving most of our data above the limit. In this regime, the altitude of the line forming region decreases with luminosity, resulting in a higher line energy with higher flux, as observed. The measured line energies also show a correlation with luminosity below this limit, but it is likely that Coulomb interactions start to decelerate the in-falling material already before the exact limit is reached.

Using Equations (51) and (58) of Becker et al. (2012), the energy-luminosity correlation follows

$$
\begin{aligned}
E_{\text {theo }}= & {\left[1+0.6\left(\frac{R_{\star}}{10 \mathrm{~km}}\right)^{-\frac{13}{14}}\left(\frac{\Lambda}{0.1}\right)^{-1}\left(\frac{\tau_{\star}}{20}\right)\left(\frac{M_{\star}}{1.4 M_{\odot}}\right)^{\frac{19}{14}}\right.} \\
& \left.\times\left(\frac{E_{\star}}{1 \mathrm{keV}}\right)^{-\frac{4}{7}}\left(\frac{L_{x}}{10^{37} \mathrm{erg} \mathrm{s}^{-1}}\right)^{-\frac{5}{7}}\right]^{-3} \times E_{\star}
\end{aligned}
$$

Here $\tau_{\star}$ is the Thomson optical depth, estimated to be around 20 for typical HMXB parameters (Becker et al. 2012). 


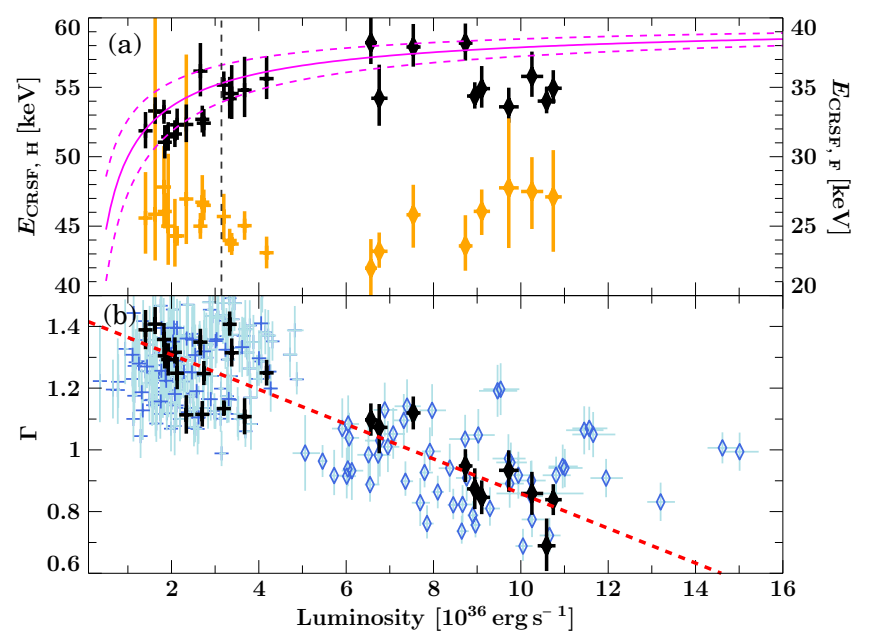

Figure 7. (a) Cyclotron line centroid energies as a function of luminosity between 3-79 keV. Black points show the energy of the harmonic line, orange points show the energy of the fundamental line, using the right-hand $y$-axis. Diamonds and crosses indicate data taken during observation I and observation II, respectively. The magenta line is the theoretical prediction for $\Lambda=1$ and $M_{\star}=1.8 M_{\odot}$, the dashed lines above and below it for $M_{\star}=1.4 M_{\odot}$ and $M_{\star}=2.2 M_{\odot}$, respectively. As can be seen, the harmonic line energies follow the prediction well and are correlated with luminosity. The black dashed lines indicates $L_{\text {coul }}$ for our set of assumptions, using a surface magnetic field of $2.59 \times 10^{12} \mathrm{G}$. For details see text. (b) Photon index $\Gamma$ as a function of X-ray luminosity. The red dashed line is a linear regression through all ks-integrated data points to guide the eye. Pulse-to-pulse data are shown in blue, ks-integrated in black.

(A color version of this figure is available in the online journal.)

This function is shown in magenta in Figure 7(a), using $\Lambda=1$ and $M_{\star}=1.8 M_{\odot}$. It describes the data of the harmonic line qualitatively well up to about $9 \times 10^{36} \mathrm{erg} \mathrm{s}^{-1}$. At this luminosity the energy of the line drops to a relatively constant level around $54 \mathrm{keV}$. The theoretically calculated energy is rather flat in this luminosity range, too, so that the discrepancy is possibly due to a constant offset, perhaps because $\Lambda$ is slightly different from 1.0.

The mass of the neutron star has a strong influence on the correlation, and we show it for two other masses, $M_{\star}=1.4 M_{\odot}$ and $M_{\star}=2.2 M_{\odot}$, as dashed magenta lines in Figure 7(a). A lower mass moves the correlation to higher energies for a given flux and results in a poorer description of the data. A mass of $M_{\star}=2.2 M_{\odot}$ provides the best description of the low luminosity data. Of course, the neutron star mass is only one of many parameters influencing this correlation, but the data seem to prefer a more massive neutron star.

Odaka et al. (2013), using Suzaku data taken in 2008, have shown that the photon index $\Gamma$ is anti-correlated with luminosity. This agrees well with the fact that the photon-index typically shows the opposite behavior as function of flux as the cyclotron line energy (Klochkov et al. 2011). As shown in Figure 7(b), our data show the same behavior, albeit with a much shallower slope. However, Odaka et al. (2013) used the NPEX continuum, which makes a direct comparison difficult. The hardening of the spectrum with X-ray flux can be understood as a direct consequence of the Coulomb shock moving further down into the accretion column where the electron gas becomes hotter. Photons can be scattered up to higher energies via the inverse Compton effect, and a harder spectrum is observed (Klochkov et al. 2011; Becker \& Wolff 2007). The pulse-to-pulse results indicate that at the highest luminosities the relation flattens out, which is very similar to what we observed for the cyclotron line energies.
With the reduced accretion radius, the local Eddington limit is also reduced. Using Equation (55) of Becker et al. (2012), we find that $L_{\text {crit }}$ is even below $L_{\text {coul }}$, i.e., Vela X-1 would be in the super-critical accretion regime. In this regime, an anticorrelation between the line energy and the luminosity would be expected, contrary to the behavior observed of the harmonic line. However, $L_{\text {crit }}$ has been calculated under the assumption that it can be scaled to the size of the accretion column compared to the surface of the neutron star. In the extreme case of a very narrow accretion column, this assumption might not hold, for example, radiation could escape through the walls of the accretion column before interacting with the in-falling material. Further calculations are needed to investigate this question.

Our model of sub-critical accretion with a narrow accretion column provides a good explanation of the measured behavior of the harmonic line. The fundamental line, however, shows a very different behavior as a function of luminosity. In fact, there seems to be an anti-correlation visible up to $7 \times 10^{36} \mathrm{erg} \mathrm{s}^{-1}$, at which point the correlation seems to flatten, i.e., it shows an opposite behavior as the harmonic line. It is difficult to conceive a physical reason for this discrepancy. Influence of the $10 \mathrm{keV}$ feature on the line energy is unlikely, as the behavior is also seen in observation II, in which the $10 \mathrm{keV}$ feature was not fitted in the ks-integrated spectra.

\subsubsection{Accretion Regime in the Off-state}

We analyzed the spectrum of the off-state during observation II. We described it with an absorbed power-law, without the need for a exponential cut-off. While the photon-index of the power-law is higher in that state, the disappearance of the cut-off makes the continuum overall harder. We could not significantly detect a cyclotron line. Doroshenko et al. (2013) analyzed a similar off-state in Suzaku data and argue that the accretion cannot be switched off completely because the spectrum is hard and residual pulsations remain visible. The luminosity was $\left(2.45_{-0.16}^{+0.19}\right) \times 10^{35} \mathrm{erg} \mathrm{s}^{-1}$ in the NuSTAR off-state data, i.e., about an order of magnitude lower than the lowest point shown in Figure 7. At that very low luminosity, it is unlikely that a shock is stopping the accretion flow, so the in-falling materials is only stopped at the surface of the neutron star. Here, the Comptonizing electron gas is hotter and denser, possibly moving the cut-off and the energy of the harmonic CRSF above $60 \mathrm{keV}$. This would be consistent with our findings of a single power-law component.

\subsection{Correlation between the Fundamental and the Harmonic Line}

The NuSTAR data allow both cyclotron lines to be simultaneously measured and their evolution to be studied on timescales as short as $1000 \mathrm{~s}$. As shown in Figure 8(a), the depth of the fundamental and the harmonic line are anti-correlated, with a Pearson's correlation coefficient of -0.68 . Spearman's rank correlation gives $\rho=-0.72$ with a false alarm probability of $3 \times 10^{-5}$, indicating a highly significant anti-correlation. Taking both $x$ - and $y$-errors into account and using the routine described by Williams et al. (2010) to fit a linear correlation, we obtain a best-fit slope of $-0.07 \pm 0.02$. As soon as the strength of the harmonic line $d_{\mathrm{CRSF}, \mathrm{H}}$ reaches $\sim 25 \mathrm{keV}$ (optical depth $\tau_{\mathrm{CRSF}, \mathrm{H}} \approx 1.34$ ), the fundamental line drops below the noise limitations of the data.

This anti-correlation is likely a result of photon-spawning. Photon-spawning occurs when an electron at an excited Landaulevel falls back to a lower state, emitting one or more photons 

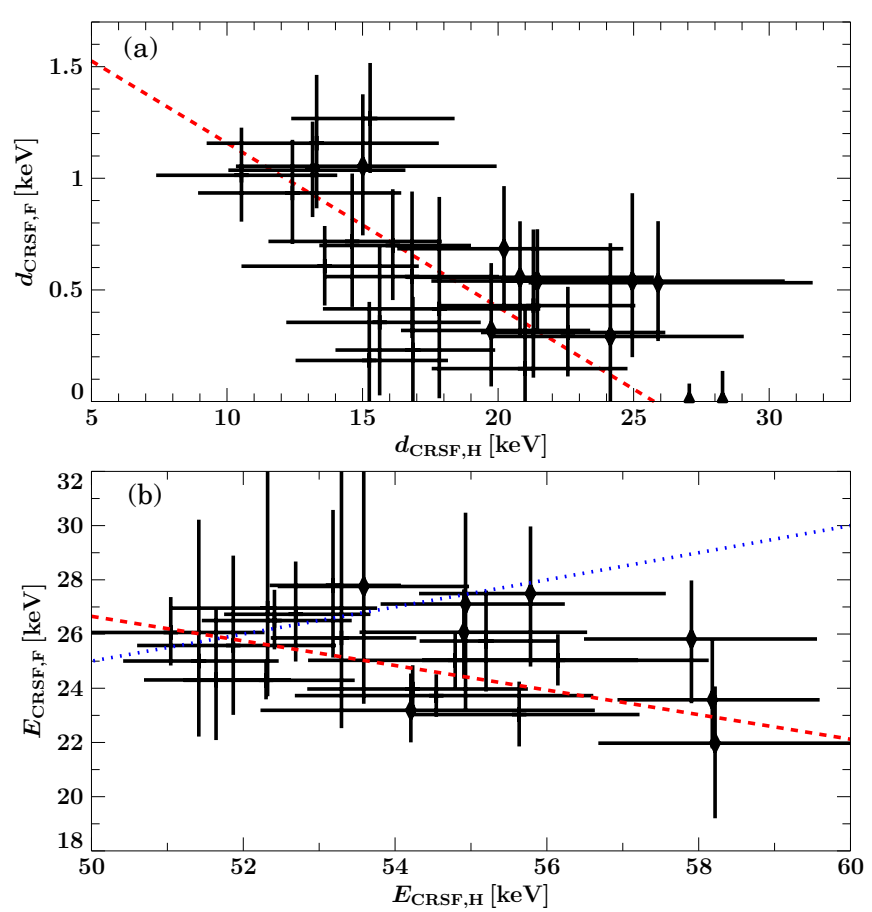

Figure 8. (a) Correlation between the depth of the harmonic CRSF $d_{\mathrm{CRSF}, \mathrm{H}}$ and the depth of the fundamental line $d_{\mathrm{CRSF}, \mathrm{F}}$. The red dashed line shows a linear fit to guide the eye. (b) Correlation between the line energies of the harmonic $\mathrm{CRSF}$ and the fundamental line. The red dashed line shows a linear fit to the data points, the blue dotted line indicates a factor of 2.0 between the line energies.

(A color version of this figure is available in the online journal.)

close to the energy of the fundamental line energy (Schönherr et al. 2007). With an increasing depth of the harmonic line, more electrons are in excited levels, resulting in a higher number of spawned photons, which can fill up the fundamental line. Photon-spawning is a direct consequence from resonant scattering and explains the observed anti-correlation of the line depths.

The strong variability of the fundamental line depth can explain apparently contradictory results in the literature, concerning the presence of the line (see, e.g., Kretschmar et al. 1997; Orlandini et al. 1998; Kreykenbohm et al. 2002; La Barbera et al. 2003; Schanne et al. 2007; Odaka et al. 2013). For example, Orlandini et al. (1998) did not find evidence for the fundamental line in BeppoSAX data. But these authors measured an equivalent width of the harmonic of $30 \pm 3 \mathrm{keV}$, much higher than our values (equivalent width of $\approx 8-14 \mathrm{keV}$ ). This prominent line could provide enough photons to fill up the fundamental line such that it is undetected. On the other hand, the optical depth $\tau$ of the line in RXTE data shown by Kreykenbohm et al. (2002) is between $0.5-1$, depending on pulse phase, close to our values of around $\tau=0.8-0.9$. In these data the fundamental line is clearly detected. We conclude that the fundamental CRSF is at $25 \mathrm{keV}$, in agreement with Kendziorra et al. (1992) but has avoided detection in some data due to photon-spawning from a prominent harmonic line.

The ratio of the energies of the cyclotron lines is shown in Figure 8(b). To first order, we would expect the line energies to be correlated, with a factor of two between the energy of the fundamental line and the harmonic line (Harding \& Daugherty 1991). The dotted line illustrates this expected correlation. Our data clearly deviate from that correlation. When ignoring the data in which the fundamental line was not detected, Pearson's correlation coefficient is -0.35 and Spearman's $\rho=-0.25$, with a false alarm probability of 0.23 . The best fit slope of a linear fit is $-0.45 \pm 0.20$. This implies an anti-correlation with a little over $1 \sigma$ confidence. Confidence contours for the time-averaged spectra show that there is no model intrinsic degeneracy present between the line energies.

Kreykenbohm et al. (2002) measure a ratio of $2.15 \pm 0.10$ between the energies of the fundamental and the harmonic line in Vela X-1, also indicating a possible deviation from the expected value of 2.0. In other sources, like 4U 1538-52 (Robba et al. 2001; Hemphill et al. 2013) and V 0332+53 (Nakajima et al. 2010) larger ratios have been observed as well. Nakajima et al. (2010) find that in V $0332+53$ the ratio increases with increasing luminosity, similar to our findings for Vela X-1. However, the shape of the fundamental line is strongly distorted in $\mathrm{V} 0332+53$, making a precise measurement of its energy difficult (Nakajima et al. 2010; Pottschmidt et al. 2005).

The deviation from the canonical ratio of 2.0 is directly connected to the different behavior as function of X-ray luminosity, as shown in Figure 7. Spawned photons from the harmonic lines are likely to influence the shape of the fundamental line, and could probably shift the measured centroid energy from its real value. The data do not provide enough resolution to investigate the shape of the fundamental line in detail. This theory can therefore only be tested by extensive theoretical calculations, which can provide insight how the line energy ratio can deviate so strongly from 2.0.

\subsection{Variability and Nature of the Absorber}

The environment around the neutron star is dominated by the heavily structured stellar wind from the optical companion. This clumpy wind is responsible for the complex absorber, which we model as partial covering, with two independent absorption columns. Additionally a photo-ionization wake is present, which moves into our line-of-sight after orbital phase 0.5 (see, e.g., Blondin et al. 1991), resulting in increased absorption at late orbital phases (Doroshenko et al. 2013, and references therein). We observe average column densities around $3 \times 10^{23} \mathrm{~cm}^{-2}$, which fit very well into that picture.

During observation I, $N_{\mathrm{H}}$ increases to a very high value of $\sim 1.2 \times 10^{24} \mathrm{~cm}^{-2}$ (see Figure 4(c)). The column density then changes smoothly over about $10 \mathrm{ks}$, decreasing again to values seen at the beginning of the observation. Assuming this event was caused by a clump moving through our line-of-sight we can calculate its size. At the orbit of the neutron $\operatorname{star}\left(R=1.7 R_{\star}\right)$, the wind has a speed of about $v_{\text {wind }}=540 \mathrm{~km} \mathrm{~s}^{-1}$ (Dupree et al. 1980). At the orbital phase of the event, $\phi_{\text {orb }}=0.68$, the wind speed is almost perpendicular to the line-of-sight, so that if the clump moves with the same velocity, it stretches out for about $r_{\mathrm{cl}}=5.4 \times 10^{11} \mathrm{~cm}$. This is about an order of magnitude larger than the clumps calculated from X-ray flares by Fürst et al. (2010) and Martínez-Núñez et al. (2013). We note that the wind speed might be drastically reduced due to the influence of the neutron star and its strong ionizing X-ray radiation (Krtička et al. 2012). However, only very close to the neutron star does the wind velocity become low enough that a clump of the size of previous works $\left(r_{\mathrm{cl}} \approx 10^{10} \mathrm{~cm}\right)$ would stay in the line-of-sight for $10 \mathrm{ks}$.

Following Fürst et al. (2010), assuming a spherical clump with $r_{\mathrm{cl}}=5.4 \times 10^{11} \mathrm{~cm}$ and an over-density of 100 compared to the smooth wind $\left(\rho_{\text {wind }}=8 \times 10^{-15} \mathrm{~g} \mathrm{~cm}^{-3}\right),{ }^{14}$ results in an absorption column of $N_{\mathrm{H}}^{\mathrm{cl}}=2 \times 10^{23} \mathrm{~cm}^{-2}$. This is about a factor

\footnotetext{
14 Note that Fürst et al. (2010) give the wrong unit for $\rho$. The correct one would be $\mathrm{kg} \mathrm{cm}^{-3}$.
} 
of 10 smaller than observed. From these estimates, it becomes clear that even an unusually large clump cannot explain the observations by simply moving through the line-of-sight with the wind speed.

There is some degeneracy between $N_{\mathrm{H}}$ and CF in the model. NUSTAR's effective area drops below $3.0 \mathrm{keV}$ so this degeneracy cannot be fully disentangled and the measured $N_{\mathrm{H}}$ values should be taken with some caution. The measured variations of $N_{\mathrm{H}}$ and $\mathrm{CF}$ are, however, larger than the model degeneracy would allow, so that the increased absorption is very likely a real event.

It is possible that the event is caused not by one clump, but by a larger number of them, moving in such a way that they start to overlap during the phase of increased absorption. According to stellar wind theories, the vast majority of the wind is located in clumps, so that many of them are close to our line-of-sight at any given moment (see, e.g., Oskinova et al. 2007). When the clumps start to overlap, the absorption column increases and at the same time the covering fraction can decrease, as observed. The assumption of spherical clumps, however, is not necessarily correct. Simulations by Dessart \& Owocki (2005), for example, show that line-driven stellar winds show complex shocked structures and not simple spherical clumps. Elongated clumps aligning with the line-of-sight could explain the observed behavior without the need of very large size. Detailed simulations would, however, be necessary to quantify this scenario.

\section{SUMMARY AND OUTLOOK}

Using two different NUSTAR observations, we performed spectral analysis with high temporal and spectral resolution. The average spectrum can be well described with a standard absorbed FDcut model. The NUSTAR data have unequivocally shown that Vela X-1 shows two cyclotron lines, with the fundamental line at about $25 \mathrm{keV}$. By performing spectral analysis on pulse-to-pulse and kilo-second time-scales, we have shown that the absorption, continuum and cyclotron line parameters are all highly variable. We showed that the strength of the fundamental line is anticorrelated with the strength of the harmonic line. This effect can be explained by photon-spawning (Schönherr et al. 2007).

For the first time, we have measured a correlation of the cyclotron line energy with luminosity. Using a surface magnetic field of $2.6 \times 10^{12} \mathrm{G}$, we can describe this correlation with the theoretical predictions of Becker et al. (2012). Assuming subcritical accretion with a Coulomb-dominated shock and a narrow accretion column, the data prefer a neutron star mass in excess of $1.4 M_{\odot}$. The mass is consistent with an independent and precise study of the optical companion and the orbit (Quaintrell et al. 2003).

Our collaboration is working on new models which will become available soon and describe the production of hard $\mathrm{X}$-rays in the accretion column based on first principle calculations, combined with the formation of cyclotron lines and the influence of the strong magnetic field on the scattering crosssections. Applying these models to Vela X-1 will allow for a description of the physical conditions inside the accretion column in more detail, and prove or disprove that Vela X-1 has a very narrow accretion column, accreting in the sub-critical regime.

This work was supported under NASA Contract No. NNG08FD60C, and made use of data from the NuSTAR mission, a project led by the California Institute of Technology, managed by the Jet Propulsion Laboratory, and funded by the National Aeronautics and Space Administration. We thank the
NuSTAR Operations, Software and Calibration teams for support with the execution and analysis of these observations. This research has made use of the NuSTAR Data Analysis Software (NuSTARDAS) jointly developed by the ASI Science Data Center (ASDC, Italy) and the California Institute of Technology (USA). We would like to thank John E. Davis for the slxfig module, which was used to produce all figures in this work. We would like to thank Fritz Schwarm for the helpful discussions about cyclotron line shapes. J.A.T. acknowledges partial support from NASA Astrophysics Data Analysis Program grant NNX13AE98G. M.B. was supported by the Centre National d'Études Spatiales (CNES). We would like to thank the anonymous referee for the useful comments.

Facility: NuSTAR

\section{REFERENCES}

Basko, M. M., \& Sunyaev, R. A. 1976, MNRAS, 175, 395

Becker, P. A., Klochkov, D., Schönherr, G., et al. 2012, A\&A, 544, A123

Becker, P. A., \& Wolff, M. T. 2007, ApJ, 654, 435

Bildsten, L., Chakrabarty, D., Chiu, J., et al. 1997, ApJS, 113, 367

Blondin, J. M., Stevens, I. R., \& Kallman, T. R. 1991, ApJ, 371, 684

Caballero, I., Kretschmar, P., Santangelo, A., et al. 2007, A\&A, 465, L21

Coburn, W., Heindl, W. A., Rothschild, R. E., et al. 2002, ApJ, 580, 394

de Kool, M., \& Anzer, U. 1993, MNRAS, 262, 726

Dessart, L., \& Owocki, S. P. 2005, A\&A, 437, 657

Doroshenko, V., Santangelo, A., Nakahira, S., et al. 2013, A\&A, 554, A37

Doroshenko, V., Santangelo, A., \& Suleimanov, V. 2011, A\&A, 529, A52

Ducci, L., Sidoli, L., Mereghetti, S., Paizis, A., \& Romano, P. 2009, MNRAS, 398, 2152

Dupree, A. K., Gursky, H., Black, J. H., et al. 1980, ApJ, 238, 969

Fürst, F., Kreykenbohm, I., Pottschmidt, K., et al. 2010, A\&A, 519, A37

Fürst, F., Suchy, S., Kreykenbohm, I., et al. 2011, A\&A, 535, A9

Fürst, F., et al. 2013, ApJ, 779, 69

Haberl, F., \& White, N. E. 1990, ApJ, 361, 225

Harding, A. K., \& Daugherty, J. K. 1991, ApJ, 374, 687

Harrison, F. A., Craig, W. W., Christensen, F. E., et al. 2013, ApJ, 770, 103

Hemphill, P., Rothschild, R., Caballero, I., et al. 2013, ApJ, 777, 61

Hiltner, W. A., Werner, J., \& Osmer, P. 1972, ApJL, 175, L19

Houck, J. C., \& Denicola, L. A. 2000, in ASP Conf. Proc. 216, Astronomical Data Analysis Software and Systems IX, ed. N. Manset, C. Veillet, \& D. Crabtree (San Francisco, CA: ASP), 591

Joss, P. C., \& Rappaport, S. A. 1984, ARA\&A, 22, 537

Kendziorra, E., Mony, B., Kretschmar, P., et al. 1992, in Proc. Yamada Conf. XXVIII, Frontiers Science Series, ed. Y. Tanaka \& K. Koyama (Tokyo: Universal Academy Press), 51

Klochkov, D., Doroshenko, V., Santangelo, A., et al. 2012, A\&A, 542, L28

Klochkov, D., Staubert, R., Santangelo, A., Rothschild, R. E., \& Ferrigno, C. 2011, A\&A, 532, A126

Kretschmar, P., Pan, H. C., Kendziorra, E., et al. 1996, A\&AS, 120, C175 Kretschmar, P., Pan, H. C., Kendziorra, E., et al. 1997, A\&A, 325, 623

Kreykenbohm, I., Coburn, W., Wilms, J., et al. 2002, A\&A, 395, 129

Kreykenbohm, I., Wilms, J., Kretschmar, P., et al. 2008, A\&A, 492, 511

Krtička, J., Kubát, J., \& Skalický, J. 2012, ApJ, 757, 162

La Barbera, A., Santangelo, A., Orlandini, M., \& Segreto, A. 2003, A\&A, 400, 993

Lamb, F. K., Pethick, C. J., \& Pines, D. 1973, ApJ, 184, 271

Larsson, S. 1996, A\&AS, 117, 197

Leahy, D. A. 1987, A\&A, 180, 275

Maitra, C., \& Paul, B. 2013, ApJ, 763, 79

Makishima, K., Mihara, T., Nagase, F., \& Tanaka, Y. 1999, ApJ, 525, 978

Martínez-Núñez, S., et al. 2013, A\&A, submitted

Mowlavi, N., Kreykenbohm, I., Shaw, S. E., et al. 2006, A\&A, 451, 187

Müller, S., Ferrigno, C., Kühnel, M., et al. 2013, A\&A, 551, A6

Nakajima, M., Mihara, T., Makishima, K., \& Niko, H. 2006, ApJ, 646, 1125

Nakajima, M., Mihara, T., \& Makishima, K. 2010, ApJ, 710, 1755

Odaka, H., Khangulyan, D., Tanaka, Y. T., et al. 2013, ApJ, 767, 70

Orlandini, M., dal Fiume, D., Frontera, F., et al. 1998, A\&A, 332, 121

Oskinova, L. M., Feldmeier, A., \& Kretschmar, P. 2012, MNRAS, 421, 2820

Oskinova, L. M., Hamann, W., \& Feldmeier, A. 2007, A\&A, 476, 1331

Owocki, S. P., Castor, J. I., \& Rybicki, G. B. 1988, ApJ, 335, 914

Palmeri, P., Mendoza, C., Kallman, T. R., Bautista, M. A., \& Meléndez, M. 2003, A\&A, 410, 359 
Pan, H. C., Kretschmar, P., Skinner, G. K., et al. 1994, ApJS, 92, 448

Pottschmidt, K., Kreykenbohm, I., Wilms, J., et al. 2005, ApJL, 634, L97

Quaintrell, H., Norton, A. J., Ash, T. D. C., et al. 2003, A\&A, 401, 313

Rivers, E., Markowitz, A., Pottschmidt, K., et al. 2010, ApJ, 709, 179

Robba, N. R., Burderi, L., Di Salvo, T., Iaria, R., \& Cusumano, G. 2001, ApJ, 562,950

Sadakane, K., Hirata, R., Jugaku, J., et al. 1985, ApJ, 288, 284

Schanne, S., Gotz, D., Gerard, L., et al. 2007, in Proc. 6th INTEGRAL Workshop; Noordwijk, ed. S. Grebenev, R. Sunyaev, \& C. Winkler (ESA SP-622: ESA Publ. Div.), 479

Schönherr, G., Wilms, J., Kretschmar, P., et al. 2007, A\&A, 472, 353

Staubert, R., Kreykenbohm, I., Kretschmar, P., et al. 2004, in 5th INTEGRAL Workshop on the INTEGRAL Universe, ed. V. Schönfelder, G. Lichti, \& C. Winkler (ESA-SP 552; Noordwijk: ESA Publ. Div.), 259
Staubert, R., Shakura, N. I., Postnov, K., et al. 2007, A\&A, 465, L25

Tanaka, Y. 1986, in Radiation Hydrodynamics in Stars and Compact Objects, Vol. 255, ed. D. Mihalas \& K.-H. A. Winkler (Berlin: Springer)

Tsygankov, S. S., Lutovinov, A. A., Churazov, E. M., \& Sunyaev, R. A. 2006, MNRAS, 371, 19

Tsygankov, S. S., Lutovinov, A. A., \& Serber, A. V. 2010, MNRAS, 401, 1628

van Kerkwijk, M. H., van Paradijs, J., Zuiderwijk, E. J., et al. 1995, A\&A, 303, 483

Verner, D. A., Ferland, G. J., Korista, K. T., \& Yakovlev, D. G. 1996, ApJ, 465,487

Watanabe, S., Sako, M., Ishida, M., et al. 2006, ApJ, 651, 421

Williams, M. J., Bureau, M., \& Cappellari, M. 2010, MNRAS, 409, 1330

Wilms, J., Allen, A., \& McCray, R. 2000, ApJ, 542, 914

Yamamoto, T., Sugizaki, M., Mihara, T., et al. 2011, PASJ, 63, 751 